\title{
A REMARK ON THE INTERSECTION OF SHELLS
}

\section{W. J. STILES}

There are many characterizations which distinguish finite-dimensional normed linear spaces from infinite-dimensional normed linear spaces. Perhaps the best known of these is the compactness of the unit ball. Recently, V. Klee [1] showed that in any infinite-dimensional normed linear space there exists a decreasing sequence of unbounded but linearly bounded closed convex sets whose intersection is empty. We will give here a somewhat similar condition which holds in all infinite-dimensional normed linear spaces but does not hold in any finite-dimensional space. We begin with the following terminology. $U$ will denote the unit ball $\{x:\|x\| \leqq 1\}$ and $S$ the unit sphere $\{x:\|x\|=1\}$. A shell will be any set of the form $\left\{x: r_{1} \leqq\|x-\alpha\|\right.$ $\leqq r_{2}$ for $\left.0 \leqq r_{1} \leqq r_{2}\right\}$ where $\alpha$ is a point.

$S$ will be called nonflat at $x$ where $x$ is in $S$ if given any open set $\theta$ containing the null vector there exists some number $\rho>1$ such that $(x+\rho U) \cap(-x+\rho U) \subset \mathcal{O}$, and $S$ will be called finitely nonflat if there exists a finite number of points $\left\{x_{j}\right\}_{j=1}^{n}$ in $S$ such that $\cap\left(x_{j}+\rho U\right) \subset \mathcal{O}$. A collection of sets has the finite intersection property if the intersection of the sets in any finite subcollection is not empty.

TheOREM. Let $B$ be a normed linear space. Then $B$ is infinite dimensional if and only if there exists a bounded collection of shells which has the finite intersection property but whose intersection is empty.

Proof. Suppose first of all that $S$ is finitely nonflat. Let $P=X\left\{R_{\alpha}: \alpha\right.$ in $\left.S\right\}$ be the product space of all real-valued functions on $S$ with the topology of pointwise convergence, and for $x$ in $U$ and $\alpha$ in $S$, let $d(x, \alpha)$ denote the norm distance from $x$ to $\alpha$. We associate with each point $x$ in $U$ the point $p_{x}$ in $P$ where $p_{x}(\alpha)=d(x, \alpha)$, and we denote the set $\left\{p_{x}: x\right.$ in $\left.U\right\}$ by $P(U)$. If $x$ and $y$ are two distinct points of $U$ and the line containing $x$ and $y$ meets $S$ at $\alpha$, then $d(x, \alpha) \neq d(y, \alpha)$. Hence, the mapping $T: P(U)$ onto $U$ defined by $T\left(p_{x}\right)=x$ is well defined. Furthermore, since $S$ is finitely nonflat, $T$ is continuous; for $T$ is clearly continuous at $p_{0}$ or $p_{x},\|x\|=1$, and continuity of $T$ can be established at all points of $P(U)$ by using a translation argument and noting the following: If $\mathcal{O}=\{x:\|x\|<r\}$ is any open ball containing the null vector and if $\left\{x_{j}\right\}_{j=1}^{n}$ are points of $S$ such that $\cap\left(x_{j}+\rho U\right) \subset \mathcal{O}$ for some $\rho>1$, then $\cap\left(t_{j} x_{j}+\left(t_{j}+2(\rho-1)\right) U\right)$

Received by the editors April 18, 1967. 
$\subset 20$ for all $t_{j}$ such that $0 \leqq t_{j} \leqq 2$. Since $P(U) \subset\{p$ in $P: 0 \leqq p(\alpha) \leqq 2\}$ and the latter set is compact by Tychonoff's theorem. $P(U)$ cannot be closed in $P$; for otherwise $U$ would be compact and $B$ would be finite dimensional. Thus there is a net $\left\{p_{\gamma}: \gamma\right.$ in $\left.\Gamma\right\}$ such that $p_{\gamma}$ is in $P(U), \lim p_{\gamma}=p$, and $p$ is not in $P(U)$. The collection of all shells of the form $\{x: p(\alpha)-1 / n \leqq\|x-\alpha\| \leqq p(\alpha)+1 / n\}$, where $\alpha$ is in $S$ and $n$ is a positive integer, has the desired properties.

If $S$ is not finitely nonflat, then for some neighborhood $\{x:\|x\| \leqq r\}$ of the null vector, the collection $\mathcal{C}$ of all balls of the form $\{x:\|x-\alpha\|$ $\leqq 1+1 / n\}$, where $\alpha$ is in $S$ and $n$ is a positive integer, has the finite intersection property. Furthermore, the intersection of any finite number of elements of $\mathcal{e}$ contains points $y$ such that $\|y\| \geqq r$. Since the intersection of all elements in $\mathcal{C}$ consists of the null vector, the collection consisting of all elements of $\mathcal{C}$ as well as the shell $\{x:\|x\|=r\}$ has the desired properties.

If $B$ is separable, $S$ is separable and the above argument can be altered slightly to yield a sequence of shells having the finite intersection property but having empty intersection. The replacement of shells by balls in the theorem is not in general possible; for example, this is prohibited in conjugate spaces by the weak* compactness of the unit ball. However, there are spaces where this replacement is possible, and J. Lindenstrauss has given in [2] necessary and sufficient conditions for the existence of a collection of balls which have the finite intersection property but have an empty intersection.

We conclude by remarking that we do not know whether the theorem is true when "shells" are replaced by "spheres" or by "spheres of radius one." ${ }^{1}$ The best result of which we are aware in this direction is the following.

THEOREM. In any infinite dimensional inner-product space $E$ there is a collection of spheres of radius one having the finite intersection property but having empty intersection.

Proof. Let $\left\{x_{r}: r\right.$ in $\left.R\right\}$ be any complete (maximal) orthogonal system in $E$ such that $\| x_{r} \mid=1 / 2$ for all $r$ in $R$. The collection consisting of all spheres of the form $S\left(x_{r}\right)=\left\{x:\left\|x-x_{r}\right\|=1\right\}$ as well as those of the form $S\left(-x_{r}\right)=\left\{x:\left\|x+x_{r}\right\|=1\right\}$, for $r$ in $R$, has the desired properties. To see this, we first note that if $y$ is in $S\left(x_{r}\right) \cap S\left(-x_{r}\right)$, then $y$ is orthogonal to $x_{r}$. Thus, if $y$ is in the intersection of all such spheres, $y$ must be orthogonal to all $x_{r}$; and by the completeness of the system, $y$ must be the null vector. But, this is impossible because

${ }_{1}$ This problem has been suggested by the referee. 
the null vector is not in any of the spheres. If $R_{n}$ is any finite subset of $R$ and $x_{\bar{r}}$ is in $R$ but not in $R_{n}$, then $3^{1 / 2} x_{\bar{r}}$ is easily seen to be contained in the set $\bigcap_{r \in R_{n}}\left(S\left(x_{r}\right) \cap S\left(-x_{r}\right)\right)$, and, therefore, the collection has the finite intersection property.

\section{REFERENCES}

1. V. Klee, $A$ note on topological properties of normed linear spaces, Proc. Amer. Math. Soc. 7(1956), 673-674.

2. J. Lindenstrauss, Extension of compact operators, Mem. Amer. Math. Soc. No. 48 (1964).

Florida State University 